\title{
Erratum to: Synthesis and investigation of silicon carbide nanowires by HFCVD method
}

S H MORTAZAVI*, M GHORANNEVISS, M DADASHBABA and R ALIPOUR

Plasma Physics Research Center, Science and Research Branch, Islamic Azad University, Tehran 009821, Iran

The original publication of this article had errors, it had to be updated and republished.

*Author for correspondence (hamideh.mortazavi@ srbiau.ac.ir) 